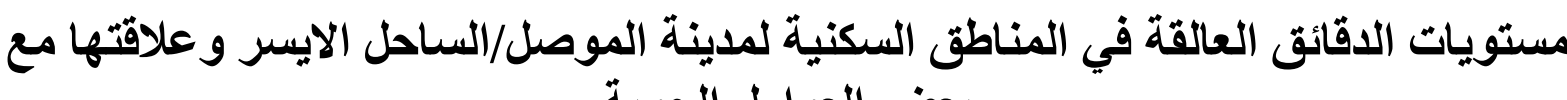
بعض العوامل الجوية

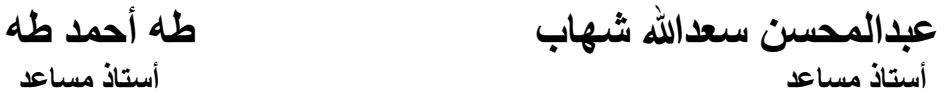

مركز بحوث البيئة والسيطرة على التلوث

جامعة الموصل

الخلاصة

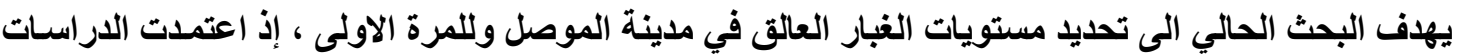

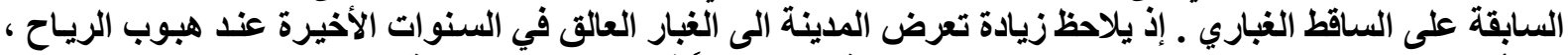

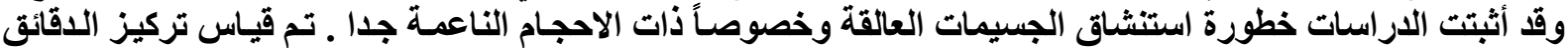

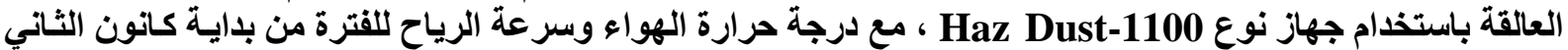

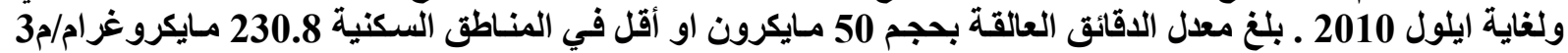

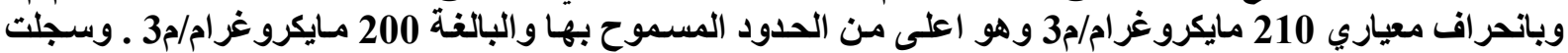

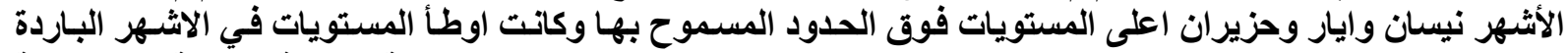

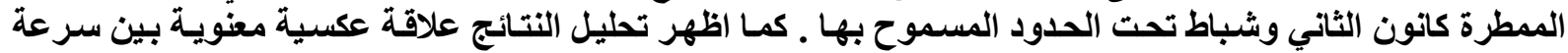

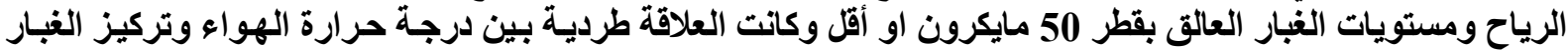

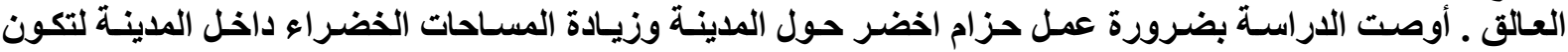
كمصدات للغبار التي تنقله الرياح .

\title{
Suspended Particulates Levels in the Left Bank Residential Areas of Mosul City and Its Relation with Some Meteorological Factors
}

Abdulmuhsin S. Shihab
Assistant Professor

Environment and Pollution Control Researches Center

\begin{abstract}
This research aimed to detect suspended particulate levels in Mosul city for the first time, as the previous studies depend on dustfall. The city was exposed in the last years to high levels of suspended particulates with the wind blowing. Many studies have found the risk of dust inhalation especially the very fine one. The measurements was conducted using Haz Dust-1100 device, including air temperature and wind speed during the study period from January to September 2010. The average suspended particulates of size 50 micron or less reached 230.8 microgram $/ \mathrm{m}^{3}$ with a standard deviation of 210 microgram $/ \mathrm{m}^{3}$ in the residential areas, which was higher than the allowable range of 200 microgram $/ \mathrm{m}^{3}$. The months April, May and June recorded the higher levels of suspended particulates above the allowable level, while the lower levels was recorded in the cold rainy months January and February. The data analysis showed a significant inverse relationship between wind speed and suspended particulate levels of 50 micron size or less and significant direct relationship with air temperature. The research recommended to make a green belt around the city and increase the green areas to decrease the suspended particulate levels.
\end{abstract}

Key Words: Suspended Particulate, Dust, Wind speed, Air temperature, Mosul, Residential

$$
\text { قبل: } 2013 \text { - } 8 \text { - } 19
$$

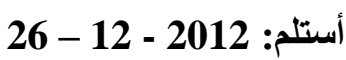




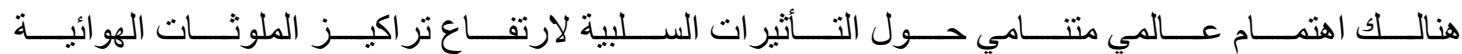

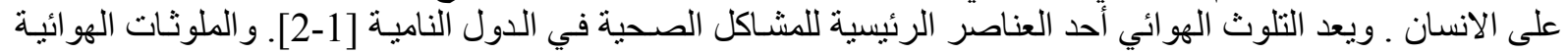

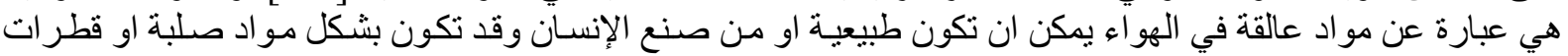

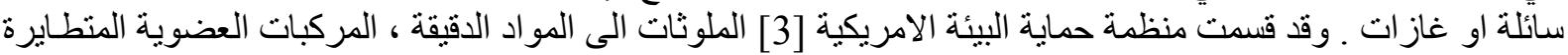

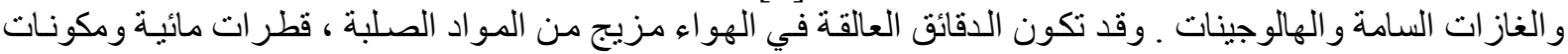

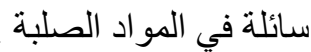

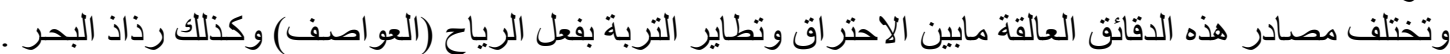

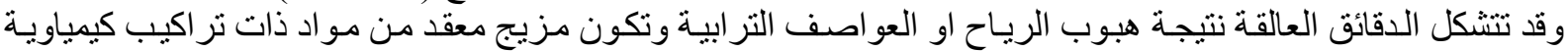

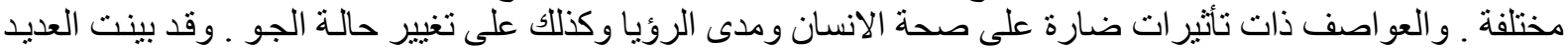

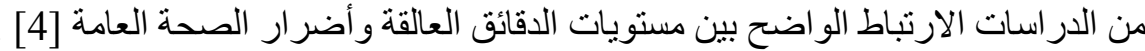

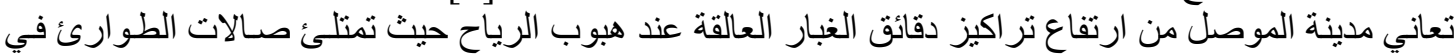

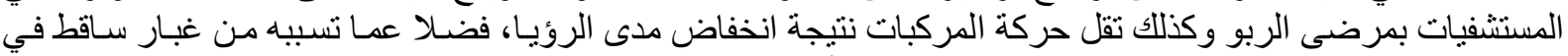

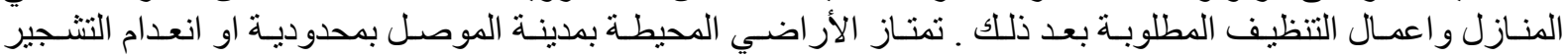

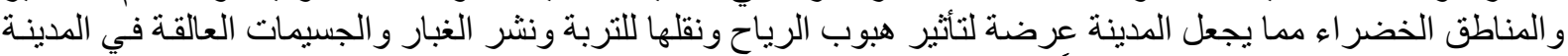

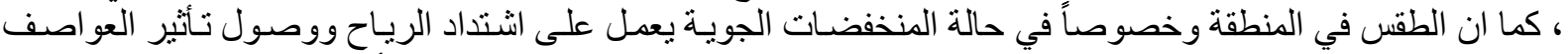

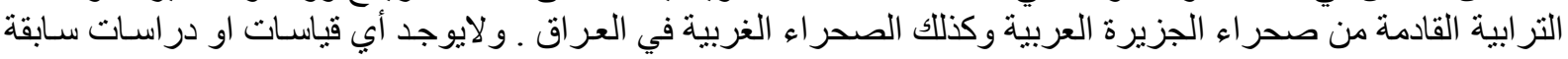

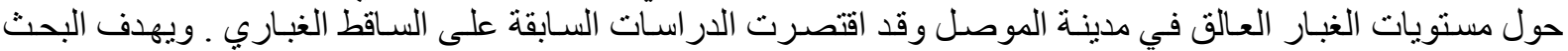

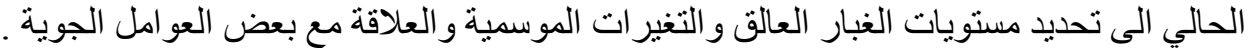

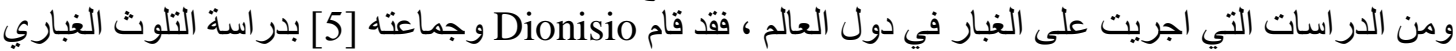

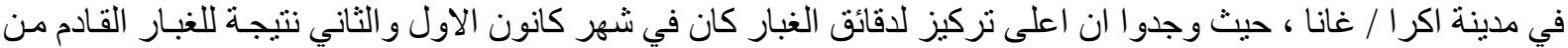

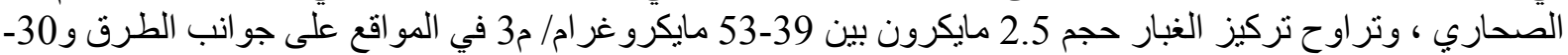

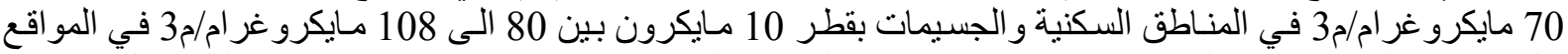

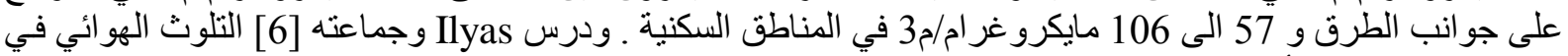

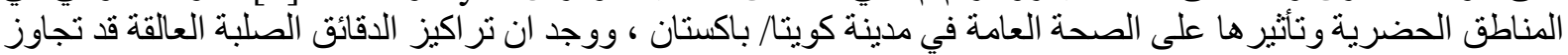

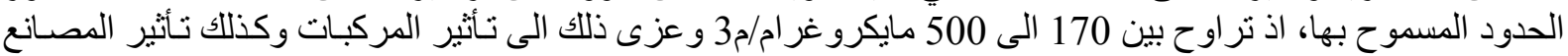

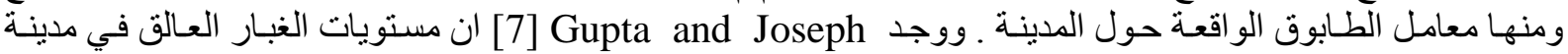

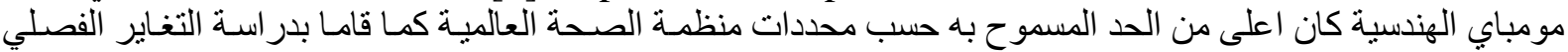

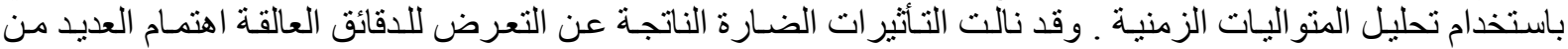

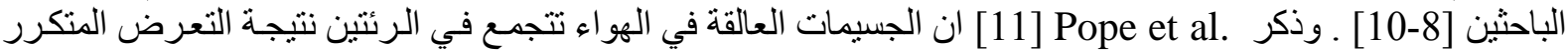
للغبار مسببة ضيق في الجهاز التنفسي ومشاكل صحية اخرى .

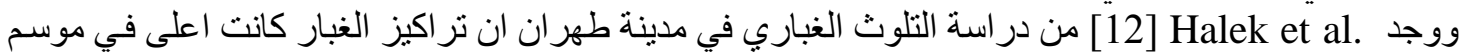

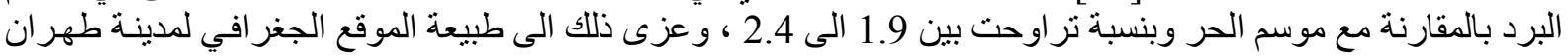

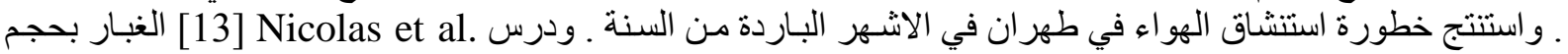

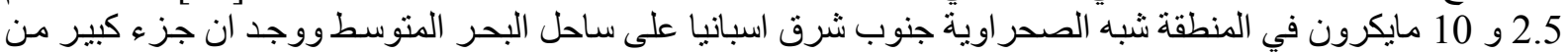

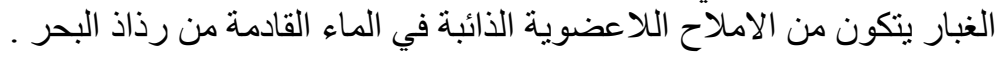

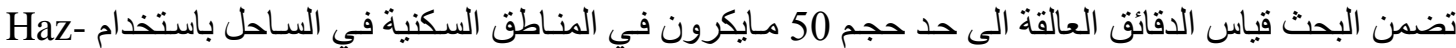
Dust-1100 Air Particulate Meter

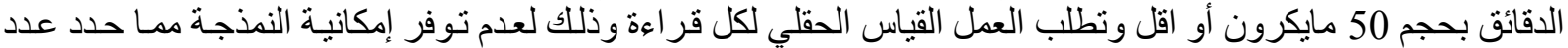

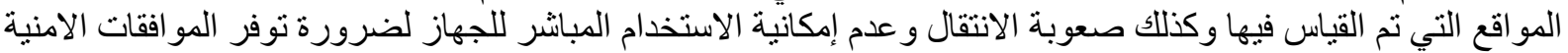

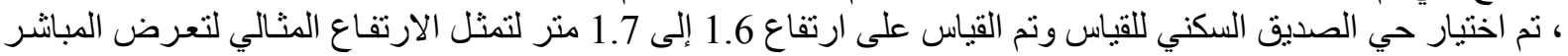

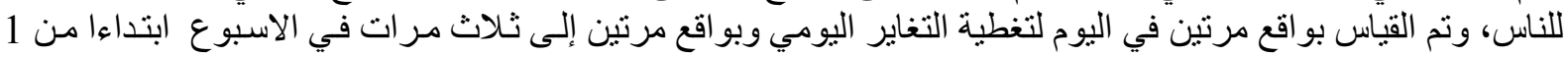

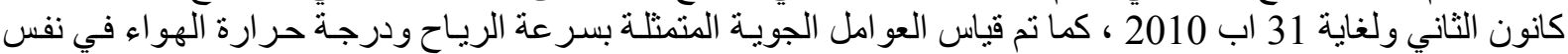
الوقت باستخدام الجهاز الملحق . 

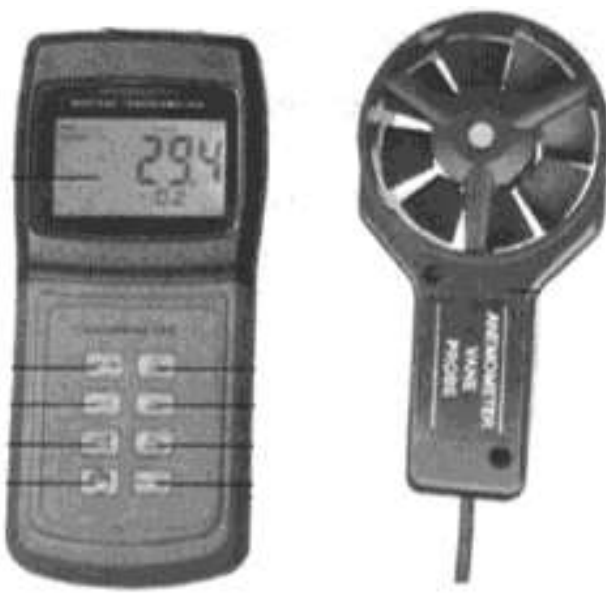

الثكل (1) جهاز قياس الدقائق العالقة لغاية حجم 50 مايكرون مع درجة الحرارة وسر عة الرياح .

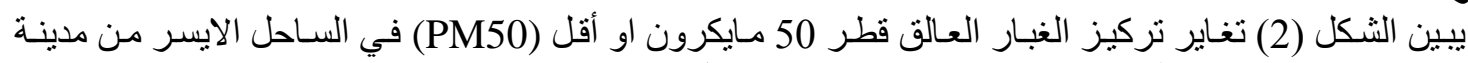

النتائج والمناقشة

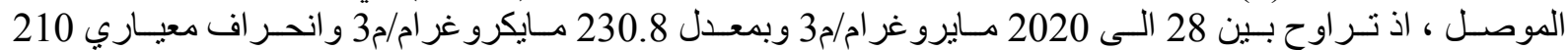

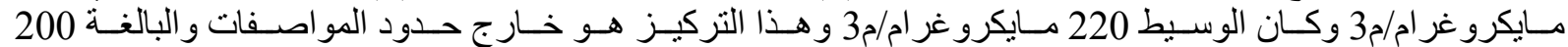

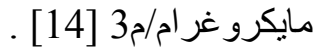

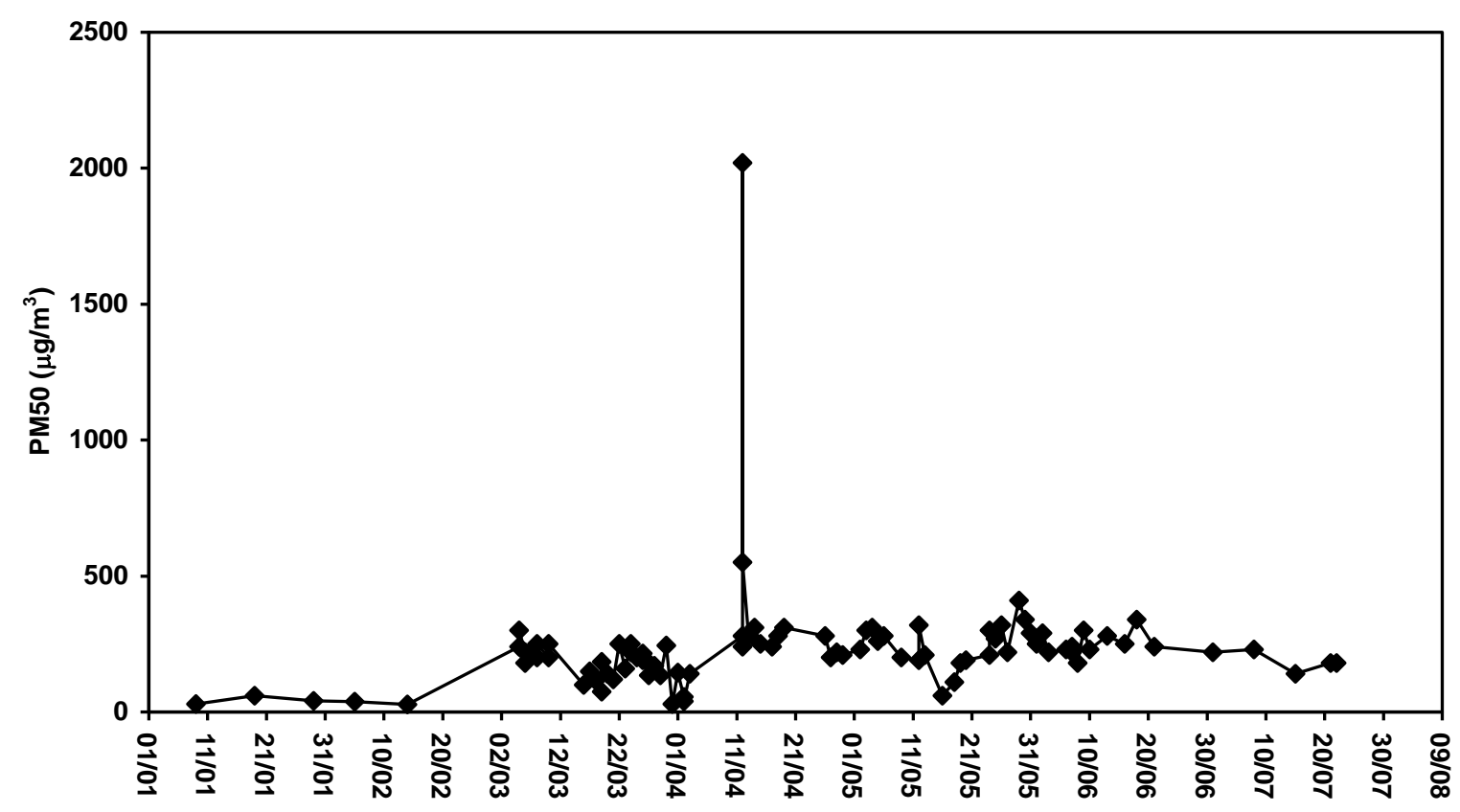

الثكل (2) تغاير تركيز الغبار العالق قطر 50 مايكرون او أقل (PM50) في الساحل الايسر من مدينة الموصل

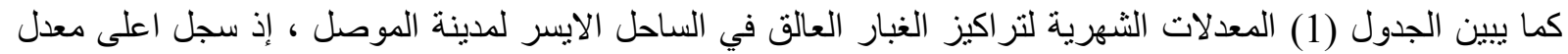

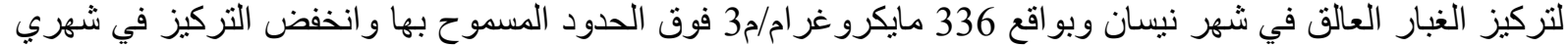

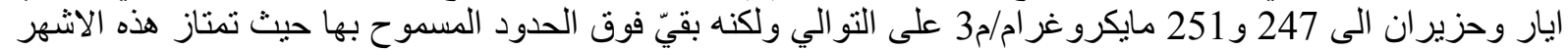



Al-Rafidain Engineering
Vol.22
No. 3
April 2014

بشدة الرياح ، في حين سجلت أوطأ التراكيز في الاشهر الباردة والرطبة كانون الثاني وشباط وبمعدل 43 و 33

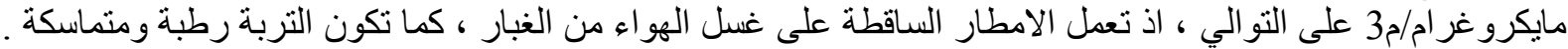

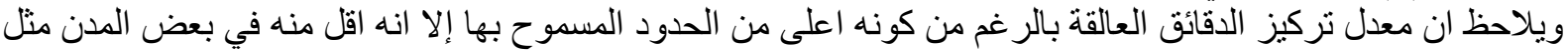

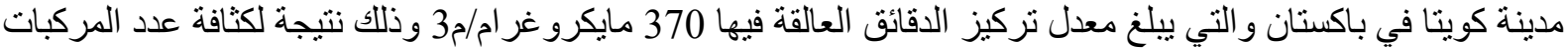

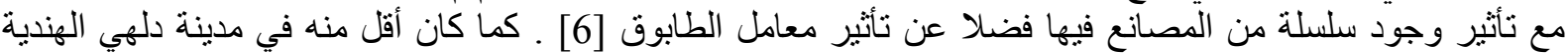

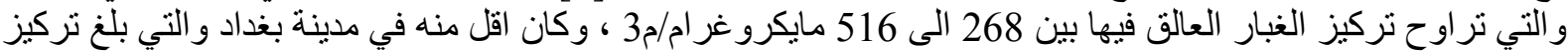
الدقائق العالقة فيها 266 مايكرو غر ام/م3 حسب المعلومات التي التي تم الحصول عليها من مديرية بيئة بغداد .

الجدول (1) تر اكيز الغبار العالق (مايكرو غر ام/23) في مدينة الموصل بحجم 50 مايكرون أو أقل خلال فترة الدراسة.

\begin{tabular}{|c|c|c|c|c|c|}
\hline Months & Mean & SD & SE & Min & Max \\
\hline Jan & 43.67 & 15.18 & 8.76 & 30 & 60 \\
\hline Feb & 33.00 & 7.07 & 5.00 & 28 & 38 \\
\hline Mar & 182.32 & 61.53 & 11.63 & 30 & 300 \\
\hline Apr & 336.11 & 434.58 & 102.43 & 40 & 2020 \\
\hline May & 247.62 & 80.55 & 17.58 & 60 & 410 \\
\hline Jun & 251.54 & 41.60 & 11.54 & 180 & 340 \\
\hline July & 190.00 & 36.06 & 16.12 & 140 & 230 \\
\hline
\end{tabular}

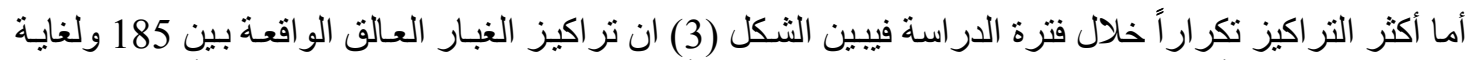

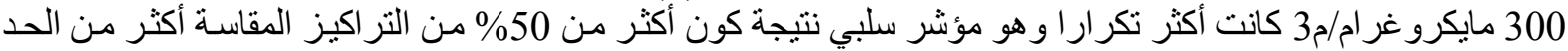

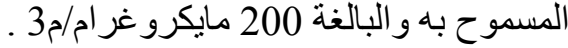

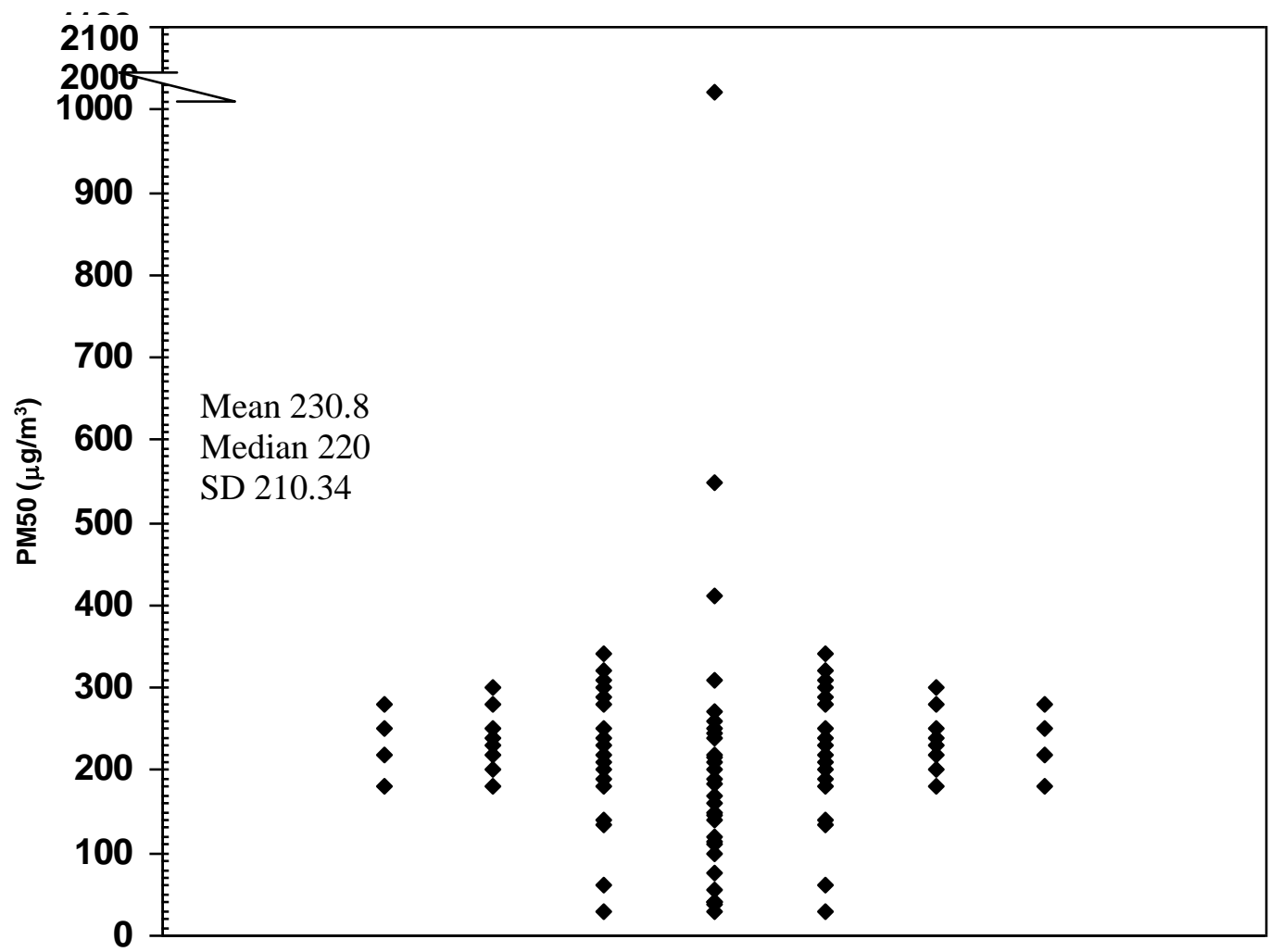

الشكل (3) مستويات الغبار العالق في مدينة الموصل موزعة حسب تكر ارها . 


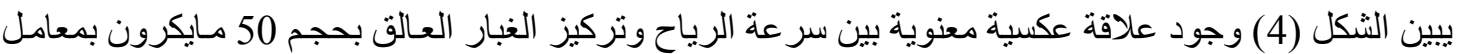

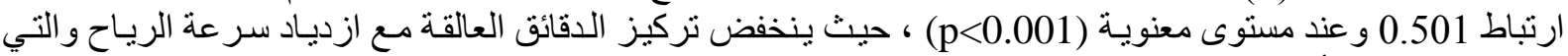

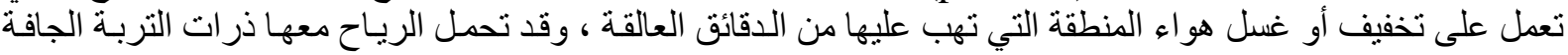

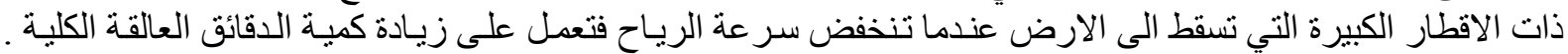

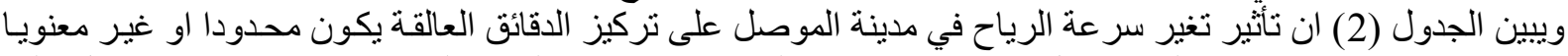

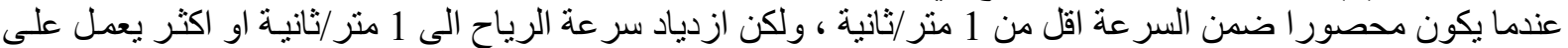

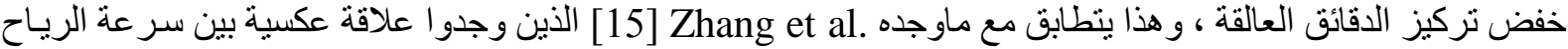

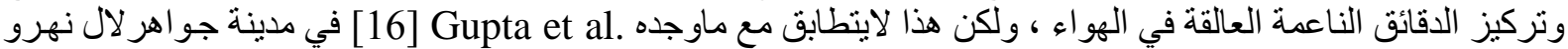

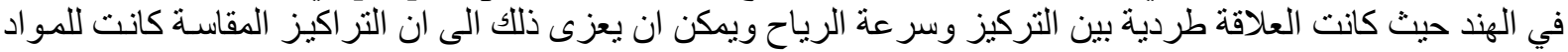

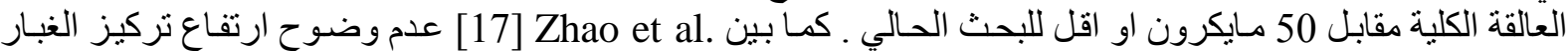

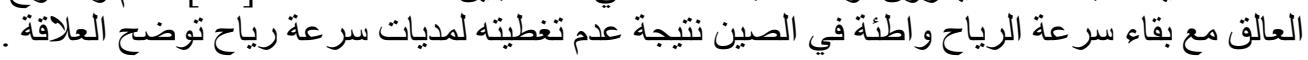

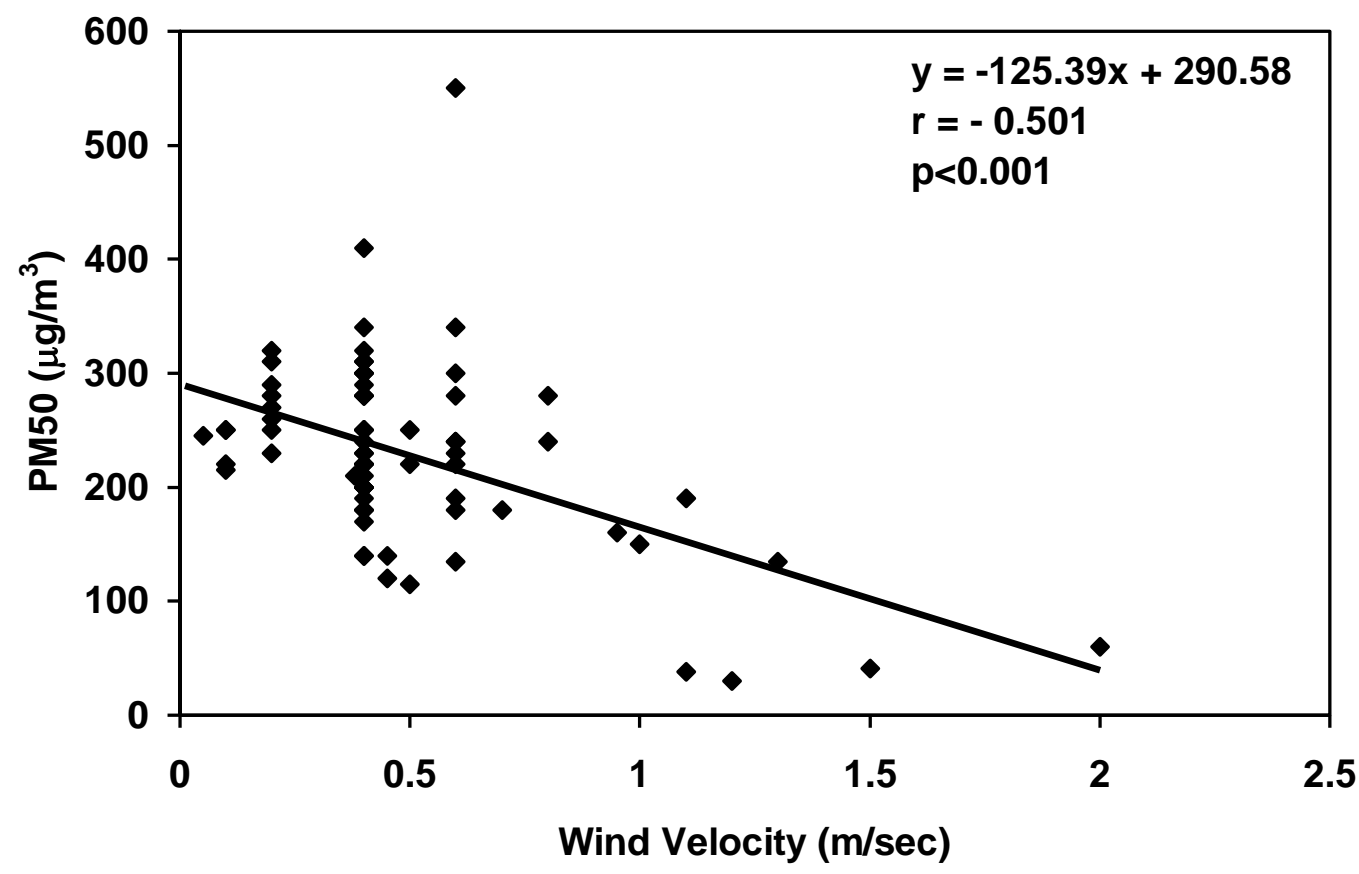

الشكل (4) العلاقة بين تر اكيز الدقائق العالقة وسر عة الرياح في مدينة الموصل .

الجدول (2) تأثثر سر عة الرياح على تر اكيز الدقائق العالق PM50 في مدينة الموصل .

\begin{tabular}{|c|c|c|c|c|c|c|}
\hline \multirow{2}{*}{ Wind speed (m/s) } & \multicolumn{5}{|c|}{ PM50 $\left(\square \mathrm{g} / \mathrm{m}^{3}\right)$} & \multirow{2}{*}{ p-value } \\
\cline { 2 - 6 } & Mean & SD & SE & Min & Max & \\
\hline$<0.25$ & $260.77 \mathrm{~b}$ & 32.33 & 8.97 & 215 & 320 & \\
\hline $0.25-0.50$ & $232.38 \mathrm{~b}$ & 64.03 & 10.12 & 115 & 410 & \multirow{2}{*}{$<0.001$} \\
\hline $0.50-0.75$ & $257.08 \mathrm{~b}$ & 108.3 & 31.26 & 135 & 550 & \\
\hline $0.75-1.0$ & $226.67 \mathrm{~b}$ & 61.10 & 35.28 & 160 & 280 & \\
\hline $1.0-2.0$ & $92.00 \mathrm{a}$ & 64.81 & 24.50 & 30 & 190 & \\
\hline
\end{tabular}

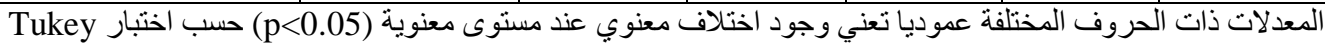

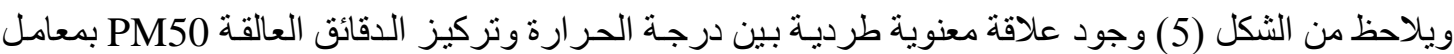

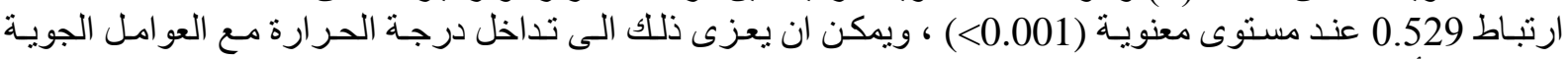

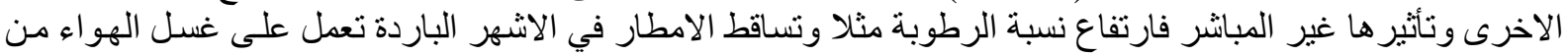

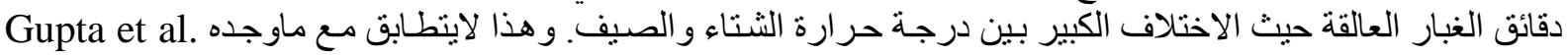

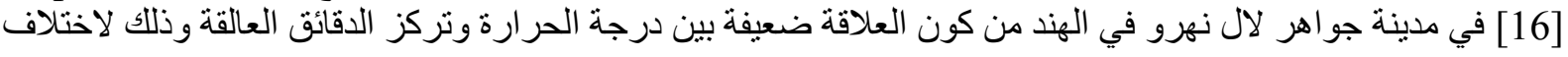



Al-Rafidain Engineering
Vol.22
No. 3
April 2014

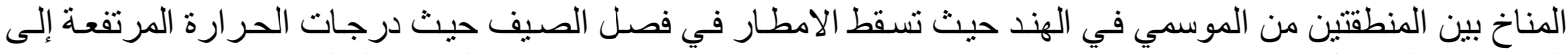

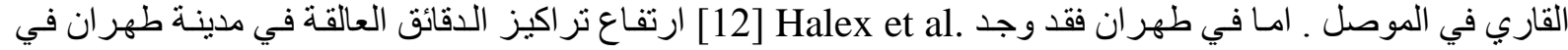

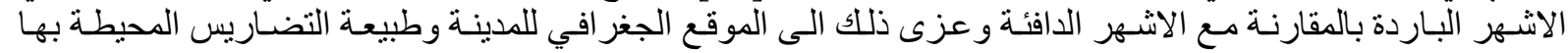

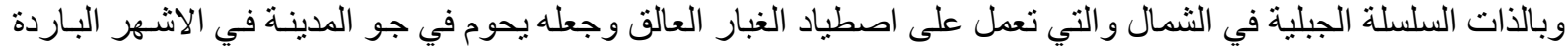
خصوصا عندما تكون الرياح ساكنة. ويلاحظ من الجدول (3) ان الفرق المعنوي في تركيز الدقائق العالقة يحدث عند درجة الإنة 20

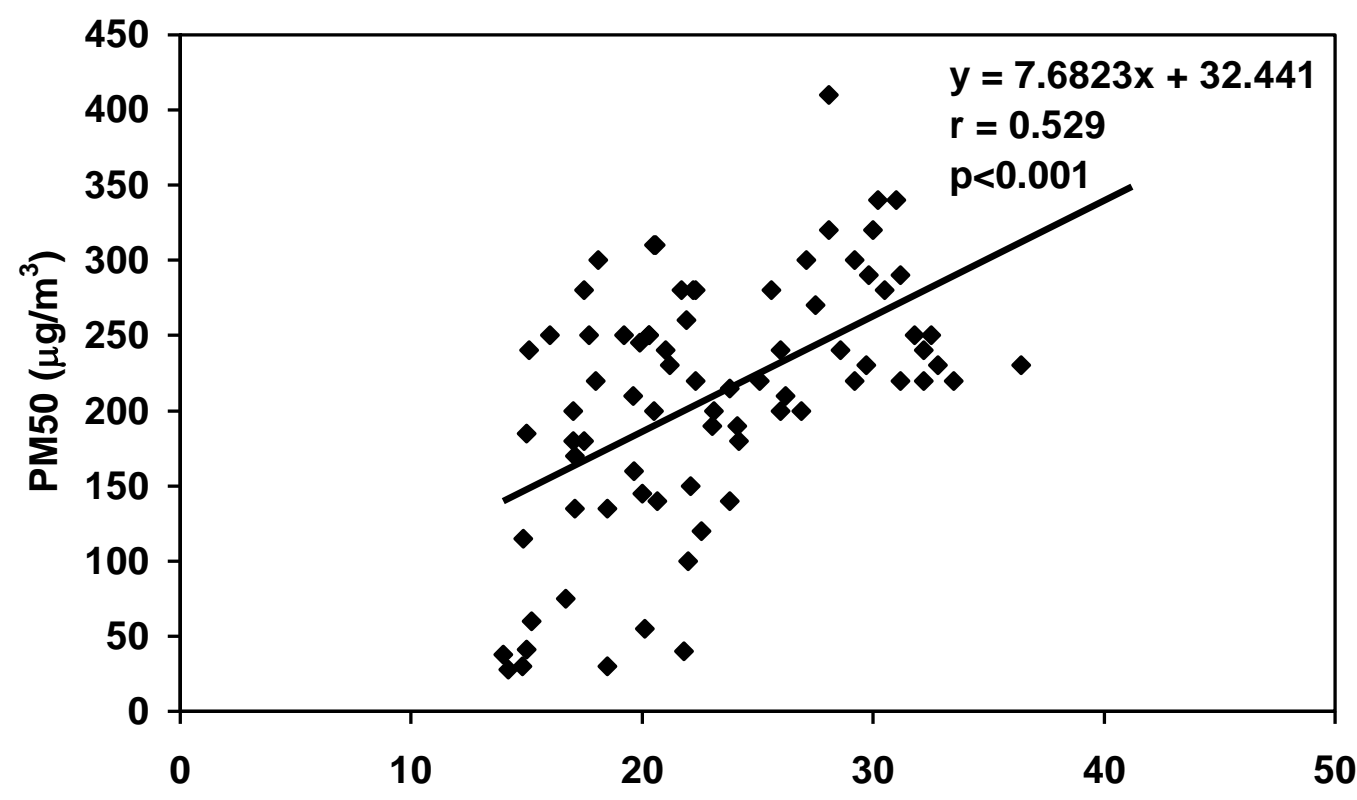

Air temperature $\left({ }^{\circ} \mathrm{C}\right)$

الثكل (5) العلاقة بين نر اكيز الدقائقَ الععالقة ودرجة حر ارة الهو اء في مدينة الموصل .

الجدول (3) تأثثر درجة حر ارة الهو اء على تر اكيز الدقائق العالق pm50 في مدينة الموصل.

\begin{tabular}{|c|c|c|c|c|c|c|}
\hline \multirow{2}{*}{ Temperature $\left({ }^{\circ} \mathrm{C}\right)$} & \multicolumn{5}{|c|}{ PM50 $\left(\square \mathrm{g} / \mathrm{m}^{3}\right)$} & \multirow{2}{*}{ p-value } \\
\hline & Mean & $\mathrm{SD}$ & SE & Min & $\operatorname{Max}$ & \\
\hline $14-20$ & $159.69 \mathrm{a}$ & 85.36 & 16.74 & 28 & 300 & \multirow{3}{*}{$<0.001$} \\
\hline $20-30$ & $227.00 \mathrm{~b}$ & 74.52 & 11.78 & 40 & 410 & \\
\hline $30-42$ & $259.17 \mathrm{~b}$ & 44.00 & 12.70 & 220 & 340 & \\
\hline
\end{tabular}

المعدلات ذات الحروف الخختلفة عموديا تعني وجود اختلاف معنوي عند مستوى معنوية (0.05>0) حسب اختبار Tukey

الاستتتاجات والتوصيات

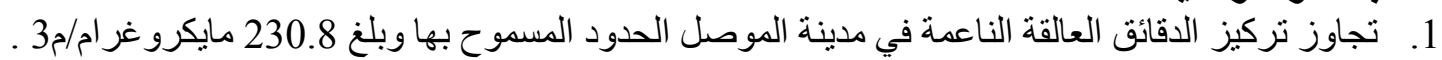

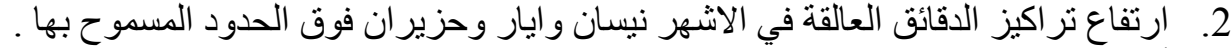

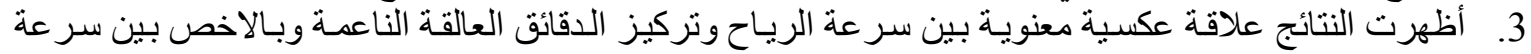

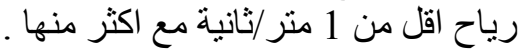
4. وجود علاقة طردية بين درجة حرارة الهواءو وتركيز الدقائق العالقة الناعمـة ، اذ انخفض تركيز الدقائق العالقة

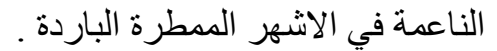

5. ضرورة انثـاء حز ام اخضر من الغابات حول مدينة الموصل وكذللك زيادة المساحات الخضر اء في المدينـة لتقليل

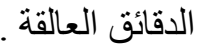


1. Brunekreef, B., Holgate, S.. Air pollution and health. Lancet, 2002, 360, 1233-1242.

2. Pope CA, Ezzati M, Dockery DW. Fine-particulate air pollution and life expectancy in the United States. N Engl J Med 2009, 360, 376-386.

3. U.S. Environmental Protection Agency. National Ambient Air Quality Standards (NAAQS); Office of Air Quality Planning and Standards, September 30, 2009.

4. Mauderly JL, Chow JC (2008) Health effects of organic aerosols. Inhal Toxicol 2008, 20, 257-288.

5. Dionisio, K.L, Arku, R.E, Hughes , AF., Vallarino , J. Carmichael, L., Spengler, JD., Agyei-mensah, S. and Ezzati M. Air Pollution in Accra /Neighborhoods: Spatial, Socioeconomic, and Temporal Patterns Environ. Sci. Technol. 2010, 44, 2270-2276.

6. Ilyas, SZ., Khattak, AI., Nasir, S.M., Qurashi, T., Durrani, R. Air pollution assessment in urban areas and its impact on human health in the city of Quetta, Pakistan. Clean Techn Environ Policy 2010, 12, 291-299

7. Gupta I, and Joseph AE. Trends of particulate matter in Mumbai city. Cheml Environ Res, 2004, 13(1\&2), 87-94.

8. Harrison, R. M., \& Yin, J. Particulate matter in the atmosphere: Which particle properties are important for its effects on health? Science of the Total Environment, 2000, 249, 85101.

9. Riesenfeld, E., Chalupa, D., Gibb, F. R., Oberdorster, G., Gelein, R., Morrow, P. E., et al. Ultrafine particle concentrations in a hospital. Inhalation Toxicology, 2000, 12, 83-94.

10. Wheeler, A. J., Williams, I., Beaumont, R. A., \& Hamilton, R. S. Characterization of particulate matter sampled during a study of children's personal exposure to airborne matter in a UK urban environment. Environmental Monitoring and Assessment, 2000, 65, 69-77.

11. Pope III, C.A., Burnett, R.T., Thun, M.J., Calle, E.E., Krewski, D., Ito, K. (2002). Lung cancer, cardiopulmonary mortality, and long-term exposure to fine particulate air pollution. Journal of the American Medical Association, 2002, 287, 1132-1141.

12. Halek F., Kianpour-Rad, M. and Kavousirahim, A. Seasonal variation in ambient PM mass and number concentrations (case study: Tehran, Iran) Environ Monit Assess 2010, 169, 501-507.

13. Nicolás, J.F., Galindo, N. Yubero, E., Pastor, C., Esclapez, R., Crespo, J. Aerosol Inorganic Ions in a Semiarid Region on the Southeastern Spanish Mediterranean Coast Water Air Soil Pollut 2009, 201, 149-159

14. NAAQS, National Ambient Air Quality Standards, Published by the Central Pollution Control Board, India, 2004.

15. Zhang, W., Xu, D., Zhuang, G., Wang, W., Guo, L. Characteristics of ambient 1-min PM2.5 variation in Beijing, Environ Monit Assess 2010, 165, 137-146.

16. Gupta AK, Patil RS, Gupta SK. A statistical analysis of particulate data sets for Jawaharlal Nehru port and surrounding harbour region in India. Environ Monit Assess, 2004, 95(1-3), 295-309

17. Zhao, Q., He, K, Rahn, KA, Ma, Y, Jia, Y, Yang, F, Duan, FLei, Y, Chen, G, Cheng, Y, Liu, H, and Wang, S. Dust storms come to Central and Southwestern China, too: implications from a major dust event in Chongqing. Atmos. Chem. Phys., 2010, 10, 2615-2630. 